\title{
THE EFFECT OF BUZZER-SHOCK UPON THE PREDICTIVE BEHAVIOR IN A TWO-CHOICE SITUATION*
}

\author{
MITSUO NAGAMACHI \\ University of Hiroshima
}

So far there are a number of articles about the predictive response in a two-choice situation. In these articles many authors reported that the asymptotic probability of the subject's predictive response of the more frequent stimulus matched the probability of its reinforcement $(2,5,6,7,8,9,10)$. This phenomenon was called the probability matching behavior and it was found with animals such as rats and fishes as well as human subjects. Estes and Straughan (5) tried to interpret the probability matching behavior according to their stimulus sampling model. In accordance with the model, under the two-choice situation of alternatives $A_{1}$ and $A_{2}$, when the relative frequency of reinforcing $A_{1}$, i. e. $\pi$, is larger than that of reinforcing $A_{2}$, i.e. $1-\pi$, the asymptotic probability of response of $A_{1}$ is equal to $\pi$ if the alternatives are exhaustive and if they are mutually exclutive. However, there are the experimental evidencies in which we could not agree to Estes' model and these results were tried to be interpreted by the secondary reinforcement model or the theory of game $(1,11)$.

The present paper is concerned with the effect of buzzer-shock given to $S$ when his response was incorrect, and with a non-fixed $\theta$ model. Estes and Burke (4) assumed that a subset of stimulus elements is sampled on each trial in an experimental situation, and that these stimulus elements are connected to one of the given responses. As a simple case it is assumed that sampling probability of stimulus elements does not vary but fixes over the whole trial. However, sampling probability of stimuli may vary on every trial. If some other conditions are given in the reinforcing situation the hypothesis of fixed sampling probability such as Estes' may not be held. For example, if some informational value is given only to $S$ 's incorrect response the effect of stimuli sampled on the incorrect trial upon the next trial response may be different from that on the correct trial under noncontingent procedure, which we designate the non-fixed $\theta$ model throughout this paper. In the next paragraph we shall describe the non-fixed $\theta$ model which will fit the experimental results.

\section{Non-Fixed $\theta$ MODEL $\star \star$}

In the two-choice situarion of noncontingent procedure the events of responses are $A_{1}$ and $A_{2}$, and the proportions of occurrence of reinforcing events $E_{1}$ and $E_{2}$ we specify $\pi$ and $1-\pi$, respectively. We shall assume the subject is in State 1 if sampled stimuli are connected to $A_{1}$ and he is in State 0 if they are not connected to $A_{1}$ in Markovian notation. Suppose some informational value is given on the trial when $S$ responds incorrectly, on which sampling probability is $\theta_{2}$, but it is $\theta_{1}$ if he does correctly. Then, the matrix of transition probabilities from one State to another can be written as follows:

* The auther is greatly indebted to professor $S$. Hayashi for valuable advice on the mathematical model, and to Mr. S. Sukemune for his editing the English phraseology.

$\star \star$ 'The derivation of equations in this paragraph was based upon the technique used in Estes' article (3). 


$$
\mathrm{Q}=\left(\begin{array}{ll}
1-\theta_{2}+\theta_{2} \pi & \theta_{2}(1-\pi) \\
\theta_{1} \pi & 1-\theta_{1} \pi
\end{array}\right)
$$

and by direct multiplication, we obtain in two-steps

$$
Q^{2}=\left(\begin{array}{cc}
\theta_{1} \pi+\theta_{1} \pi\left(1-\theta_{2}-\theta_{1} \pi+\theta_{2} \pi\right) & 1-\theta_{1} \pi-\theta_{1} \pi\left(1-\theta_{2}-\theta_{1} \pi+\theta_{2} \pi\right) \\
+\left(1-\theta_{2}-\theta_{1} \pi+\theta_{2} \pi\right)^{2} & -\left(1-\theta_{2}-\theta_{1} \pi+\theta_{2} \pi\right)^{2} \\
\theta_{1} \pi+\theta_{1} \pi\left(1-\theta_{2}-\theta_{1} \pi+\theta_{2} \pi\right) & 1-\theta_{1} \pi-\theta_{1} \pi\left(1-\theta_{2}-\theta_{1} \pi+\theta_{2} \pi\right)
\end{array}\right)
$$

and in general, we can write down

$$
\mathrm{Q}^{n}=\left(\begin{array}{ll}
\pi_{\theta}+\left(1-\pi_{\theta}\right)\left(1-\theta_{\pi}\right)^{n \cdot} & \left(1-\pi_{\theta}\right)\left[1-\left(1-\theta_{\pi}\right)^{n}\right] \\
\pi_{\theta}\left[1-\left(1-\theta_{\pi}\right)^{n}\right] & 1-\pi_{\theta}\left[1-\left(1-\theta_{\pi}\right)^{n}\right]
\end{array}\right)
$$

where

$$
\pi_{\theta}=\theta_{1} \pi /\left(\theta_{2}+\theta_{1} \pi-\theta_{2} \pi\right)
$$

and

$$
\theta_{\pi}=\theta_{2}+\theta_{1} \pi-\theta_{2} \pi
$$

If he shall define $q_{1}^{(n)}$ and $q_{0}^{(n)}$ to be the probabilities of State 1 and State 0 after $n$ trials and $q^{(n)}$ to be

$$
q^{(n)}=\left(q_{1}^{(n)}, q_{0}^{(n)}\right) .
$$

By direct multiplication we can write that

$$
q^{(1)}=q^{(0)} \mathrm{Q}
$$

and by mathematical induction we can derive

$$
q^{(n)}=q^{(0)} \mathrm{Q}^{n}
$$

Assuming $p_{n}$ the probability of $A_{1}$ response on trial $n$ is equal to the probability of sampled elements conditioned to $A_{1}$ on trial $n-1$, then

$$
\begin{aligned}
p_{n} & =\pi_{\theta}-\left(\pi_{\theta}-p_{1}\right)\left(1-\theta_{\pi}\right)^{n-1} \\
& =\frac{\theta_{1} \pi}{\theta_{2}+\theta_{1} \pi-\theta_{2} \pi}-\left(\frac{\theta_{1} \pi}{\theta_{2}+\theta_{1} \pi-\theta_{2} \pi}-p_{1}\right)\left(1-\theta_{2}-\theta_{1} \pi+\theta_{2} \pi\right)^{n-1} .
\end{aligned}
$$

From (8), after large $n$ trials the asymptotic probability of $A_{1}$ response is

$$
p_{\infty} \simeq \theta_{1} \pi /\left(\theta_{2}+\theta_{1} \pi-\theta_{2} \pi\right) \text {. }
$$

In the above derivations, we assumed that sampling probability on $S$ 's incorrect trial is different from that on $S$ 's correct trial. But now, if we assume there is no difference be ween there two sampling probabilities, that is, $\theta_{1}=\theta_{2}=\theta$, we can change equation (8) to

$$
p_{n}=\pi-\left(\pi-p_{1}\right)(1-\theta)^{n-1},
$$

and this equation is one derived from Estes' model.

In general, we usually represent the learning process with the average percentage of a given response per block of trials. Therefore, we must transform (8) to the equation to be able to represent it per block. Assuming the number of trials of a block $k$, we can write the response probability in block $m$

$$
P_{m}=\pi_{\theta}-\frac{1}{k \pi_{\theta}}\left(\pi_{\theta}-P_{1}\right)\left(1-\theta_{\pi}\right)^{k^{(m-1)}}\left[1-\left(1-\theta_{\pi}\right)\right]
$$

and assuming the initial response probability in block $1 P_{1}$, we can derive 


$$
P_{m}=\frac{\theta_{1} \pi}{\theta_{2}+\theta_{1} \pi-\theta_{2} \pi}-\left(\frac{\theta_{1} \pi}{\theta_{2}+\theta_{1} \pi-\theta_{2} \pi}-P_{1}\right)\left(1-\theta_{2}-\theta_{1} \pi+\theta_{2} \pi\right)^{k(m-1)} .
$$

We shall assume that some informational value (for example, a buzzer-shock as in the following experimentation) is added only to $S$ 's incorrect responses and that it is not to $S$ 's correct responses. Therefore, $\theta_{1}$ must be equal to $\theta$ in Estes' model since $\theta_{1}$ is sampling probability in the case of $S$ 's correct responses to which no other conditions are given. We can get the parameter value ${ }^{\star}$ of $\theta_{1}$ or $\theta$ from the results of usual two-choice experiments without any specific conditions. Then, if we get the parameter value of $\theta_{2}$, we can calculate $P_{m}$ from (10). To get $\theta_{2}$ value, we sum $P_{m}$,

$$
\sum_{m} P_{m}=r \pi_{\theta}-\left(\pi_{\theta}-P_{1}\right) \frac{\left[1-\left(1-\theta_{\pi}\right)^{k r}\right]}{1-\left(1-\theta_{\pi}\right)^{k}} .
$$

However, since $k r$ is very large, $\left(1-\theta_{\pi}\right)^{k r}$ almost equals to zero. Therefore,

$$
\begin{aligned}
\sum_{m} P_{m} & =r \pi_{\theta}-\left(\pi_{\theta}-P_{1}\right) \frac{1}{1-\left(1-\theta_{\pi}\right)^{k}} \\
& =\frac{r \theta_{1} \pi}{\theta_{2}+\theta_{1} \pi-\theta_{2} \pi}-\left(\frac{\theta_{1} \pi}{\theta_{2}+\theta_{1} \pi-\theta_{2} \pi}-P_{1}\right) \frac{1}{1-\left(1-\theta_{2}-\theta_{1} \pi+\theta_{2} \pi\right)^{k}} .
\end{aligned}
$$

Then, if we get $\theta$ from Estes' model, we can get the value of $\theta_{2}$ from (11) and we may compare the predicted learning curve with the empirical data.

Here, it will be necessary to consider the properties of $\theta_{1}$ and $\theta_{2}$. If $\theta_{1}=\theta_{2}=\theta$, $\mathrm{P}_{\infty}$ becomes equal to $\pi$ asymptotically. This result agrees to the conclusion of asymptotic probability of response in Estes' model in which sampling probability of stimulus is $\theta$. If $\theta_{1}$ is larger than $\theta_{2}$, assuming $\theta_{1}-\theta_{2}=\varepsilon_{1}>0$,

$$
P_{\infty}=\frac{\theta_{1} \pi}{\theta_{1}-(1-\pi) \varepsilon_{1}}>\pi
$$

since $\theta_{1}-(1-\pi) \varepsilon_{1}<\theta_{1}$. Therefore, when the effect of $\theta_{2}$ is smaller than $\theta_{1}$ in the experimental situation, the asymptote of $S$ 's response probability will tend to be larger than the probability of reinforcement $\pi$. If $\theta_{1}<\theta_{2}$, similarly

$$
P_{\infty} \simeq \frac{\theta_{1} \pi}{\theta_{1}+(1-\pi) \varepsilon_{2}}<\pi
$$

assuming $\theta_{2}-\theta_{1}=\varepsilon_{2}>0$. In the experimental condition with larger value of $\theta_{2}$ than $\theta_{1}$ the asymptote of response probability will tend to be lower than $\pi$.

Thus, in the present model, it is dependent upon the additional conditions in the experimentation whether the asymptotic probability of $A_{1}$ response is higher, or lower than $\pi$, or to be equal.

\section{METHOD}

Subjects - The $S$ s were 20 male and female undergraduate students at the University of Hiroshima. They were divided into two groups, an experimental group of $9 S$ s and a control group of 11 Ss. Ss received

$\star$ To obtain $\theta_{1}$ value from the model, from $\left(8^{\prime}\right)$ we can write

and from $\left(11^{\prime}\right)$ we can get the $\theta_{1}$ value.

$$
\sum_{m} P_{m}=r \pi\left(\pi-P_{1}\right) \frac{1}{1-(1-\theta)^{k}}
$$




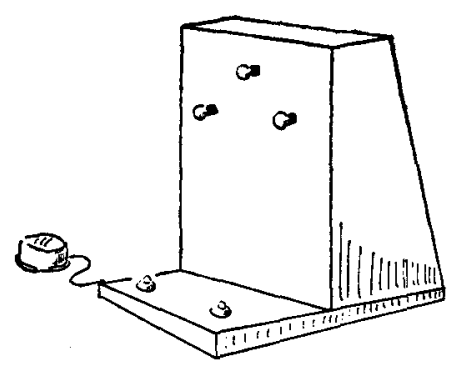

Fig. 1 Apparatus

an individual test in a semi-dark room. All $S$ s had no experience in this sort of the experiment.

Apparatus-The apparatus consisted of a black standing L-type panel-board of the Humphreys type (See Figure 1). Three light lamps mounted on the $181 / 4 \times 10 \mathrm{in}$. standing panelboard which was placed upon a table. A red lamp was set up on the midline, $2 \frac{1}{2}$ in. from the top of the panel, which was used as a signal. Other two white lamps were $4 \mathrm{in}$. apart, $2 \mathrm{in}$. under the red lamp, which were used as reinforcing events. $S$ was required to guess which lamp was lit on a given trial. There were two keybuttons, $4 \mathrm{in}$. apart and $3 \mathrm{in}$. from the standing board, mounted on the horizontal panel-board, $181 / 4$ in. $\times 4 \frac{1}{2}$ in., in front of $S$. It was implied that pressing of the right key mean the prediction of the right lamp on the standing panel-board to turn on and that pressing of the left key meant the prediction of the left lamp. After $S$ pressed either the right or the left key, one of two lamps turned on, which reinforced S's guessing. A 6-v. buzzer was used for buzzershock given to $S$ if he guessed incorrectly. It was held by $S$ 's left hand over the trials in order to strengthen shock ${ }^{\star}$ or noxious effect to be given to $S$. The signal lamp was lit for $2 \mathrm{sec}$. and after the rest period of $2 \mathrm{sec}$. one of reinforcing lamps was lit for 2 sec. $S$ must guess which lamp turned on before the reinforcing event occured. The intertrial interval was $4 \mathrm{sec}$. These time intervals and duration of the buzzer sound were controlled automatically behind the apparatus and a screen was set such that $S$ could not see its control.

Procedure - After all $S$ s were explained about the apparatus they were given an instruction in that $S$ s must guess, by pressing one of keys, which lamp will be lit on each trial and that they must respond correctly and try to get as many correct responses as they could. Ss in the experimental group received buzzer-shock when they guessed incorrectly but $S$ s in the control group did not. About half of the $S$ s in each group were run under the condition of $75 \%$ reinforcement for the right lamp and the other about half under the condition of $75 \%$ for the left lamp. It was determined randomly which lamp turned on on each trial in consideration that a reinforcing event occured in $75 \%$ per 20 trials, and that the same event did not continue to occur more than six times. $S$ received a total of 200 trials without pause.

\section{Results AND Discussion}

Learning curves - The average percentages of responses to the more frequent stimulus in blocks of 20 trials were presented in Figure 2. Learning curves in experimental and control conditions are similar up to 120 trials but the distinction between the two groups appears in block 7 and it tends to be large towards the last block. Figure 2 does not show the smooth learning curves in both conditions. This would result in inappropriate stimulus sequence presented in blocks 3 and 6 since two curves are very similar with each other.

In the control condition we assume $\theta_{1}$ and $\theta_{2}$ equal to $\theta$ in Estes' model be-

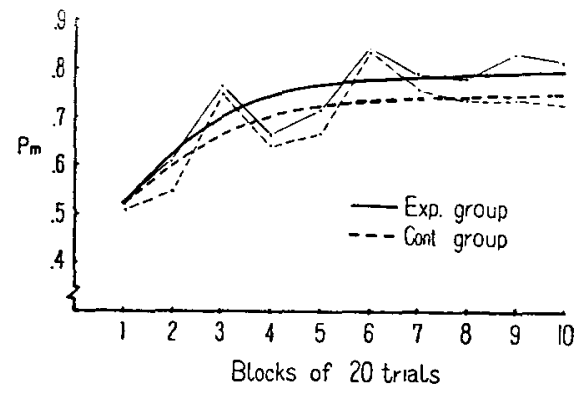

Fig. 2 Empirical and theoretical curves. cause no additional information was given to this group. Then, we can calculate $\theta$ from $\left(11^{\prime}\right)$ and we obtained .026 . By substituting $\theta$ value into (8), and by calculating $P_{m}$, we had

* It may not be appropriate to use the term of "shock" here. However, the author used term, shock, throughout this paper since all $S$ s reported that they had the noxious effect by the sound and the vibration of the buzzer. In the paragraphs to follow, the terms "shoce" and "buzzer-shock" will be used interchangeably. 
a smooth learning curve (a dotted line in Fig. 2). Since $S$ s in the experimental group received buzzer-shock when they guessed incorrectly, we shall assume $\theta_{1}>\theta_{2}$. But it is assumed that $\theta_{1}$ equals to $\theta$ since $S$ s were given no special information concerning the reinforcing light when they guessed correctly. We can obtain $\theta_{2}$ value from (11) by substituting $\theta_{1}$ $=.026, P_{1}=.510$, and $\pi=.75$. We verified the assumption $\theta_{1}>\theta_{2}$ since $\theta_{2}$ was $.020 . \mathrm{A}$ smooth curve in Fig. 2 is the theoretical learning curve in the experimental condition.

The asymptote.-We often deal with a model in the asymptotic level when we discuss the evaluation of the model. The average response probability of the more frequent stimulus during the last 80 trials was .740 in controls, and the theoretical asymptote was $\pi=.75$. As shown in Table 1 , the average response probability during the last 80 trials in the experimental group was .801 and the theoretical asymptote $P_{\infty}$ was .796 obtained from (9). The $t$ values for differences between observed and theoretical values in two groups seem satisfactorily low.

Table 1. Predicted and Observed Response Probabilities of $A_{1}$ during the Last 80 Trials.

\begin{tabular}{l|c|cc|c}
\hline Group & Observed & Predicted & t & df \\
\hline Exp. & .801 & .796 & 0.09 & 8 \\
Cont. & .740 & .750 & 0.18 & 10 \\
\hline
\end{tabular}

The Mann-Whitney $U$ Test shows that the difference between two groups in terms of the asymptotic response probability during the last 80 trials is significant at the .05 level. Therefore, we can say that the effect of buzzer-shock upon $S$ 's choices resulted in increasing the proportion of choosing the more frequent stimulus.

In this experiment, the buzzer-shock given to the incorrect responses may operate to inhibit $S$ s to make incorrect responses, especially much more to inhibit incorrect responses of the less frequent stimulus.

We made an attempt to quantify the behavior effected by the shock, assuming that the sampling probability of stimulus elements depends upon the presence or absence of the shock. The experimental results support the proposed model with respect to the asymptotic level. However, we must say this model is a special one because we, in general, assume that the sampling probability of elements may vary on each trial in acquisition.

\section{SUMMARY}

This paper is concerned with the effect of the buzzer-shock upon the guessing behavior in a two-choice situation. Buzzer-shock was given to $S s$ in an experimental group when they guessed incorrectly. The acquisition trials were 200 trials. Ss were 20 undergraduate students.

After 121 trials the differences between the experimental group and the control group (without buzzershock) were found, and the asymptotic probability of guessing the more frequent stimulus was significantly different between the two groups. It was concluded that buzzer-shock for incorrect responses tends to increase their choices of the more frequent stimulus.

The non-fixed $\theta$ model was applied to the data, in which it was assumed that the sampling probability of stimulus elements depends upon the presence or absence of the shock. The present model seems to fit the experimental findings with respect to the asymptotic response level. 


\section{REFERENCES}

1. Bush, R.R. \& Wilson, T.R. Two-choice behavior of paradise fish. J. exp. Psychol., 1956, 51, 315-322.

2. Estes, W. K. Individual behavior in uncertain situation. In Thrall, Coombs, and Davis (Eds.) Decision Processes, New York: JohnWiley. 1954.

3. Estes, W.K. Component and pattern models with Markovian interpretations. In Bush and Estes (Eds.) Studies in Mathematical Learning Theory. Standford: Stanford Univ. Press. 1959.

4. Estes,W.K. \& Burke, C. J. A theory of stimulus variability in learning. Psychol. Rev., 1953, 60, 276-286.

5. Estes, W. K. \& Straughan, J. H. Analysis of a verbal conditioning situation in terms of statistical learning theory. J. exp. Psychol., 1954, 47, 225-234.

6. Goodnow, J. J. Determinants of choice-distribution in two-choice situations. Amer. J. Psy- chol., 1955, 68, 106-116.

7. Grant, D. A., Hake, H, W., \& Hornseth, J. P. Acquisition and extinction of a verbal conditioned response with differing percentages of reinforcement. J. exp. Psychol., 1951, 42, 1-5.

8. Hake, H.W. \& Hyman, R. Perception of statistical structure of a random series of binary symbols. J. exp. Psychol., 1953, 45, 64-74.

9. Humphreys, L.G. Acquisition and extinction of verbal expectations in a situation analogous to conditi oning. J. exp. Psychol., 1939, 25, 294301.

10. Jarvik, M. E. Probability learning and a negative recency effect in the serial anticipation of alternative symbols. J. exp. Psychol., 1951, 41, 291-297.

11. Siegel, S. \& Goldstein, D. A. Decision-making behavior in a two-choice uncertain outcome situation. J. exp. Psychol., 1959, 57, 37-42.

MS. received VII 10,60 .

Mitsuo Nagamachi (長町三生, 1936-) graduated from Univ. of Hiroshima in 1958, and obtained M. A. in 1960. 1960- Graduate student in predoctoral course, Univ. of Hiroshima. Specialized fields: Mathematical learning theory and memory. Article; A mathematical model of retention (in Japanese). Jap. Psychol, Rev., $1959,3,100-115$. 